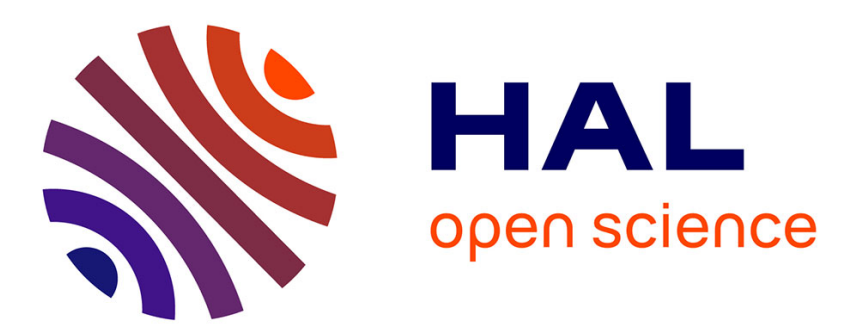

\title{
Multipath routing for high-data rate applications in ZigBee wireless sensor networks
}

\author{
Zahia Bidai, Moufida Maimour
}

\section{To cite this version:}

Zahia Bidai, Moufida Maimour. Multipath routing for high-data rate applications in ZigBee wireless sensor networks. 6th International Conference on New Technologies, Mobility \& Security, NTMS'2014, Mar 2014, Dubai, United Arab Emirates. hal-01087938

\section{HAL Id: hal-01087938 \\ https://hal.science/hal-01087938}

Submitted on 27 Nov 2014

HAL is a multi-disciplinary open access archive for the deposit and dissemination of scientific research documents, whether they are published or not. The documents may come from teaching and research institutions in France or abroad, or from public or private research centers.
L'archive ouverte pluridisciplinaire HAL, est destinée au dépôt et à la diffusion de documents scientifiques de niveau recherche, publiés ou non, émanant des établissements d'enseignement et de recherche français ou étrangers, des laboratoires publics ou privés. 


\section{Multipath Routing for High-Data Rate Applications in ZigBee Wireless Sensor Networks}

\author{
Zahia Bidai \\ Laboratory of Research in Industrial \\ Computing and Networking, LRIIR \\ Oran University, BP 1524 El M'naouar, Algeria \\ bidai.zahia@univ-oran.dz
}

\author{
Moufida Maimour* \\ *Université de Lorraine, CRAN, UMR 7039 \\ Campus Sciences, BP 70239 \\ Vandœuvre-lès-Nancy Cedex, 54506, France \\ ${ }^{\dagger}$ CNRS, CRAN, UMR 7039, France \\ moufida.maimour@univ-lorraine.fr
}

\begin{abstract}
In this paper, we propose $Z M R$ (ZigBee Multipath Routing), a novel hybrid multipath routing protocol for ZigBee/IEEE 802.15.4 networks. The main goal is to improve the global throughput which is basically insufficient in ZigBee-based Wireless Sensor Networks to satisfy high bandwidth requirements for high traffic applications like structure health monitoring systems, human health care systems and multimedia surveillance systems. The solution is based on the parent-child links and the availability of hierarchical address allocation process of the cluster-tree topology and the neighboring links. We compare our proposed multipath routing to the ZigBee one-path hierarchical tree routing $T R$ through detailed simulations which showed that the proposed multipath routing enhances application performance in terms of effective bandwidth needed for high data rate applications, packet delivery ratio, incurred delay and network lifetime.
\end{abstract}

Index Terms-IEEE 802.15.4/ZigBee, Cluster-tree Topology, ZigBee Multipath Routing, Tree Routing, Wireless Sensor Networks.

\section{INTRODUCTION}

Wireless sensor networks (WSNs) have appeared as one of the emerging technologies that combine automated sensing, embedded computing and wireless networking into tiny embedded devices. While the early research on WSNs has mainly focused on monitoring applications [1][2] based on low-rate data collection, current WSN applications can support more complex operations like building automation, industrial automation [3] and personal health care [4]. Besides these, the availability of low-cost CMOS cameras and microphones where a tiny sensor can be equipped with modules for collecting visual and audio informations [5][6], have enabled a new class of WSNs: multimedia or visual wireless sensor networks (WMSN/WVSN) [7] which has significantly enhance a wide range of applications like object detection, recognition, tracking and surveillance, etc. What is common in these emerging application domains is that performance and quality of service (QoS) assurance are becoming crucial as opposed to the besteffort performance in traditional monitoring applications.

The importance of WSN has been enforced by the delivery of the most popular IEEE 802.15.4/ZigBee technology. ZigBee aims at handling low data rate, low cost devices and long-life batteries making it very suitable for wireless sensor networks [8].
However, ZigBee technology suffers from its limited bandwidth $(250 \mathrm{kbps}$ at $2.4 \mathrm{GHz})$ and extending it to meet requirements of high data rate applications such as multimedia ones is a real challenge. Few works [9][10][11][12] already considered multimedia and high data applications over IEEE 802.15.4 at PHY and MAC layers.

Routing is a key process to consider in WSNs when dealing with QoS requirements as routing decisions can impact the network lifetime, packet delivery rates and end-to-end packet delays. Zigbee specification [13] provides basic recommendations on how to perform routing tasks. However, these are based on a simplified version of AODV and Tree Routing $[14][15][16][17]$ that do not comply with the high data and multimedia application requirements. Having only one available route means that, in high data rate application scenarios with varying radio channel characteristics, connectivity is likely to be lost. Indeed, node/link failure causes packets to be dropped and may cause a delay in delivering the data to the destination in time; thus the requirements of these applications are not met which often making this approach to routing unsuitable for such applications. It is desirable to find alternative methods of reducing the effect of node/link failure on performance. Motivated by this, the aim of the work presented here is to promote the use of WSN ZigBee in high data rate applications by designing an efficient multipath algorithm. It is based on the parent-child relationships of the ZigBee cluster-tree topology and the neighboring links of the ZigBee mesh topology where direct transmissions between non parent-child devices is allowed. Multipath routing is desirable because an increase in the number of possible routes provides load balancing and increases the robustness and the bandwidth requirement of the application [18][19]. Also, since there is more bandwidth available, a smaller end-to-end delay can be achieved [20]. Given the considerable advantages of the multi-path routing technique, we focus on guaranteeing the required soft quality of service through multipath routing. To the best of our knowledge, there has been no earlier work that deals with handling multipath routing in ZigBee WSN on top of the tree-based topology.

This paper is the extension of the authors previous publications [21] [22]. The new contributions of this paper consist of presenting an enhanced ZigBee multipath routing 
to improve the QoS metrics required by the high data rate applications such as multimedia applications. The hybrid nature of the multipath routing proposed allows to achieve this purpose. Firstly, The proactive part of the routing protocol is activated in order to reduce the end-to-end latency and to increase the network bandwidth, the reactive part of the protocol is triggered to discover other node disjoint paths. Extensive simulations have been carried out to evaluate the improved multipath routing from different aspects include the offered multipath effective bandwidth. More details will be given later.

This paper is structured as follows. Section II briefly introduces the IEEE 802.15.4/ZigBee standard and mainly the cluster-tree topology and Tree Routing protocol. Our proposed ZigBee multipath routing protocol is described in Section III and Section IV provides our simulation results. Finally, Section V concludes the paper.

\section{IEEE 802.15.4 AND ZIGBEE}

We consider a WSN using the ZigBee technology, composed of static sensor nodes deployed in a field of interest. ZigBee is a robust wireless communication standard managed by the ZigBee Alliance [13] and based on the IEEE 802.15.4 physical and MAC layer standard [23]. It defines a network layer, application framework as well as security services. The MAC layer defines two types of nodes: Reduced Function Devices (RFDs) and Fully Functional Devices (FFDs).

The ZigBee network layer defines how a network address is assigned to each participant ZigBee device and how a data packet is routed. ZigBee specification extends the basic star topology of an IEEE 802.15.4 [23] to a mesh or a cluster-tree topology. In a cluster-tree topology, the root, called the ZigBee coordinator (ZC), and all internal nodes refered to as ZigBee Routers (ZRs) are FFDs. RFDs can only be leaf nodes and are designated by ZigBee End Devices (ZEDs).

A cluster-tree ZigBee network is characterized by three topological parameters $\left(L_{m}, C_{m}, R_{m}\right)$ fixed by the ZigBee coordinator where $L_{m}$ is the maximum depth of the tree, $C_{m}$ and $R_{m}$ are respectively the maximum number of joining children (ZEDs or ZRs) and ZRs a parent can have. According to ZigBee specification, the $\mathrm{ZC}$ is at depth 0 and the end devices are at depth $L_{m}$ and can only be ZEDs. Based on its depth $d\left(d \in\left[0, L_{m}-1\right]\right)$ in the tree, a (ZC or ZR) assigns for every joining ZR a range of consecutive addresses with size $C \operatorname{skip}(d)$. Assume that for a given parent node at depth $d$ with network address $A_{p}$, the addresses in $\left[1, R_{m}\right]$ and $\left[R_{m}+1, C_{m}\right]$ are assigned to its children ZRs and ZEDs respectively, then the $k^{t h} \mathrm{ZR}$ and the $n^{\text {th }} \mathrm{ZED}$ will be assigned the network addresses :

$$
\begin{gathered}
A_{k}=A_{p}+C \operatorname{skip}(d) \cdot(k-1)+1, \quad k \in\left[1, R_{m}\right] \\
A_{n}=A_{p}+C \operatorname{skip}(d) \cdot R_{m}+n, \quad n \in\left[1, C_{m}-R_{m}\right]
\end{gathered}
$$

$C \operatorname{sip}(d)$ is given by :

$$
\operatorname{Cskip}(d)= \begin{cases}1+C_{m} \cdot\left(L_{m}-d-1\right) & \text { if } R_{m}=1 \\ \frac{C_{m} \cdot R_{m}^{\left(L_{m}-d-1\right)}+R_{m}-C_{m}-1}{R_{m}-1} & \text { otherwise }\end{cases}
$$

Two routing protocols are considered in ZigBee depending on the adopted topology. The table-driven routing (similar to AODV : Ad hoc On demand Distance Vector) [24][25][26][27] is used in the mesh topology while tree routing (TR) [15][14] is adopted for a tree topology. $T R$ can directly infer the routing paths from network addresses based on the parentchild relationships. No extra memory and broadcast overhead are required. However, routing paths could be longer because the data packets follow the hierarchical tree topology to the destination even if the destination is close to the source node. When a packet is received by a ZigBee router (ZR), it decides on whether the next hop node for the destination address is up or down the tree. Since $T R$ is simple to implement and is lighter in terms of memory and processing requirements than the AODV routing, it is more suitable for the ZigBee limited resources devices.

\section{ZigBeE MULTIPATH ROUTING}

Our proposal consists in $Z M R$ (ZigBee Multipath Routing), a multipath routing protocol built on top of the ZigBee $T R$ protocol. On one hand, we take advantage of $T R$ 's simplicity and limited required resources. On the other hand, the single path $T R$ 's failure drawback can be avoided since other alternative paths can be used. Indeed, in $Z M R$, more than one path (if available) are used simultaneously, so the aggregate bandwidth may satisfy the bandwidth requirement of high data rate applications. Moreover, these paths are node disjoint which allows additional resources and higher fault-tolerance. In what follows, we begin by giving some preliminaries before presenting how $Z M R$ builds its node disjoint paths.

\section{A. Preliminaries}

In this work, we only consider the case of routing toward the Sink (a central controller or a gateway) of the WSN. The cluster-tree topology is assumed to be rooted at the Sink that plays the role of the coordinator. The $Z M R$ is applied on a steady state network where all the devices are well associated to their parent devices. To make its forwarding decisions, $Z M R$ relies on this tree structure mainly on the already parent-child established relationships in addition to neighborhood relationships. In $Z M R$, each node is assumed to maintain an up-to-date neighbors table that contains all nodes that are physically in its radio range (parent, child or adjacent nodes). This neighbors table can be built simply during joining process when a node scans its neighborhood to find potential parent. This information is used during the routing task. We further assume that neighboring links are symmetric. That is, if node $N_{a}$ is a neighbor of node $N_{b}$ then node $N_{b}$ is a neighbor of node $N_{a}$.

In order to be able to check, based only on parent-child relationships, if two paths are node disjoint or not, we introduce the ZigBee Tree Path information. For a given node $C$ 
at depth $d_{c} \in\left[1, L_{m}\right]$, the ZigBee Tree Path information of node $C$ noted $Z T P_{c}$ is an integer sequence $\left(Z_{1}^{c}, Z_{2}^{c}, \ldots, Z_{d_{c}}^{c}\right)$ that defines the parent-child path in the tree from the Sink to $C$. Each element $Z_{k}^{c}\left(1 \leq k \leq d_{c}\right)$ is simply the rank of the child node located at depth $k$ in the path from the Sink to $C$.

The value of $Z_{k}^{c}$ components of the ZigBee tree path information can be computed using :

$$
Z_{k}^{c}=\left\lfloor\frac{A_{c}-k-\sum_{i=1}^{k-1} C \operatorname{skip}(i-1) \cdot\left(Z_{i}^{c}-1\right)}{C \operatorname{skip}(k-1)}\right\rfloor+1
$$

where $A_{c}$ is the network address of $C$ and can be deduced using the recursive form of (1) as follows :

$$
\begin{aligned}
A_{c}= & \overbrace{\underbrace{\left(d_{c}-1\right)}+\sum_{i=1}^{d_{c}-1} \operatorname{Cskip}(i-1) \cdot\left(Z_{i}^{c}-1\right)}^{A_{p} \text { of } C} \\
& +C \operatorname{skip}\left(d_{c}-1\right) \cdot\left(Z_{d_{c}}^{c}-1\right)+1
\end{aligned}
$$

Note that the first value $Z_{1}^{c}$ of the ZigBee tree path information of node $C$ represents the tree branch number to which belongs the node $C$, where a branch is a subtree rooted at the child number $Z_{1}^{c}$ of the PAN coordinator (Sink). Therefore, two different nodes $N_{a}$ and $N_{b}$ belong to two different subtrees if and only if their first values $Z_{1}^{a}$ and $Z_{1}^{b}$, respectively, are different. In this case, we say that the two parent-child paths of $N_{a}$ and $N_{b}$, respectively, are two node disjoint paths.

\section{B. Multipath Routing Algorithm}

$Z M R$ is a hybrid node disjoint multipath routing. When the source detects an event and in order to reduce the end-to-end latency, it starts immediately transmitting data packets to the Sink using the proactive hierarchical routing $T R$ where the parent-child path is already established during the association phase at the MAC layer. Also, it starts the route discovery phase which represents the reactive part of $Z M R$. Unlike the traditional discovery phase with its heavy traffic of control messages (for instance RREQs), in our case, a light discovery phase is used in order to estabilish additional paths based on the parent-child and neighboring links and the ZigBee Tree Path information.

Given a source node $S$, the basic idea to build subsequent paths is as follow: Initialy, the source chooses as the nexthop node an adjacent neighbor from its neighbors table. If the neighbor is the Sink, noted by $N S$, or a node which belongs to a branch (subtree) not already used by $T R$, noted by $N T R$, then a new path of one link (in the case of $N S$ neighbor) or composed of one neighbor link and the parent-child links from $N T R$ to the Sink, can be directly used without triggering the discovery process. Otherwise, the source sends an Explore message Explore Msg which is forwarded (unicast and never broadcast) from node to node using forwarding decisions. Whenever a node forwards the Explore Msg, it records the next node and the node from which it has receveid the Explore $M s g$ in its routing table.

When an intermediate node $C$ at depth $d_{c}$ receives Explore $M s g$, it starts searching for the next-hop node among its neighbors. If more than one candidate neighbor exist then the one with the smallest depth is chosen. Three cases are considered :

The intermediate node $C$ has a $N S$ or a $N T R$ neighbor node, in this case, the Explore $M s g$ is not fowarded and the new disjoint path established is the one followed by the Explore Msg concatenated to either (i) the last link between $C$ and the Sink, or (ii) the tree path from the NTR node to the Sink, respectively. In this case, $C$ node sends a ResponseMsg message towards the source using the reverse path already established during the discovery phase.

Based on the depth of the first common node between the $Z T P_{c}$ of node $C$ and the $Z T P_{Z_{1}^{c}}$ of the node which has firstly used $T R$ routing in $C^{\prime}$ branch, node $C$ chooses the next-hop node, two cases are possible :

- The first common node is at depth $\left(d_{c}-1\right)$. This common ancestor is not considered as the next-hop node and an adjacent node $N$ is chosen from the neighbors table of node $C$.

- The first common node is at a depth less than $\left(d_{c}-1\right)$. In this case, $T R$ is applied by the intermediate node $C$.

The intermediate node $C$ has no candidate node as its nexthop node. It sends a Error $M s g$ message to its predecessor node. This latter, tries to forward Explore $M s g$ to a qualified node among its neighbors; otherwise, in its turn, it sends an Error $M s g$ to its predecessor. This feedback mechanism continues until an intermediate node finds a good candidate node to forward the Explore Msg or the ErrorMsg arrives at the source. In this case, if another candidate node is available and a new disjoint path is required, then the source can initiate a novel discovery process.

When the source node receives the Response Msg from its neighbor on which the discovery path was initiated, it means that another valid disjoint path is established and the source can use it for transmitting its data packets.

We assume that the source has adjacent nodes (are not parent, child, Sink and NTR neighbors) and sends Explore Msg in order to discover subsequent disjoint paths. Algorithm 1 describes the discovery process at the intermediate node $C$.

Illustrative Example Figure 1 shows a network topology with parameters $\left(L_{m}, C_{m}, R_{m}\right)=(3,4,4)$. Where all the sensor nodes are evenly distributed on a square-shaped area. The Sink is the $Z C$ located at the center. The transmission range $(T r)$ chosen in this topology allows to cover at least three neighbors and at most eight neighbors. The $\operatorname{Cskip}(d)$ values are respectively 21,5 and 1 at depth $d$ where $d=0,1,2$ (Equation 3). For a given node, the two numbers between brackets are respectively the address (that results from equation 1) and the $C \operatorname{skip}(d)$ value. The other numbers denote only addresses of nodes (equation 1 ). In this example, the source node $s$ is at depth 2 and has address 17 and nodes $a, c, b, f$ and $k$ as neighbors (one parent neighbor and four adjacent neighbors). By applying equation 4, Its $Z T P_{s}=(1,4)$ (node $s$ is the fourth child of the node $a$, 


\begin{tabular}{l}
$\overline{\text { Algorithm } 1 \text { Discovery process at an intermediate node } C}$ \\
\hline Inputs :
\end{tabular}

$P$ : this node parent, $C:$ the current node, $N:$ the adjacent neighbor node of $C$.

$N S$ : the adjacent neighbor of $C$ is the Sink.

$N T R$ : the adjacent neighbor of $C$ is the node which belongs to a subtree (branch) not already used by $T R$.

$F_{1}^{a}$ : the node which has firstly used the $T R$ routing in branch number $Z_{1}^{a}$ of node $A$.

$Z T P_{F_{1}^{a}}$ : the ZigBee tree path information of node $F_{1}^{a}$.

Intersection $\left(Z T P_{a}, Z T P_{b}\right)$ returns the rank of the first different element between $Z T P_{a}$ and $Z T P_{b}$.

Inclusion $\left(Z T P_{a}, Z T P_{b}\right)$ test if the node $B$ belongs to the parent-child path of $A$.

\section{Output :}

ExploreMsg.X : a field in the ExploreMsg packet that contains the next-hop node.

1: search in the neighbors table of $C$ for a candidate neighbor $N$

2: if ( $N$ is a $N S$ or a $N T R$ ) then

3: $\quad$ update the routing table of $C$; send ResponseMsg packet to the source over the reverse path;

4: else

5: $\quad \mathrm{t}=$ Intersection $\left(Z T P_{F_{1}^{c}}, Z T P_{c}\right)$

6: $\quad$ if $\left(t=d_{c}\right)$ then

7: $\quad$ the first common node is at depth $\left.d_{c}-1\right\}$

8: $\quad$ search in the neighbors table of $C$ for an adjacent node $N$ such that Inclusion $\left(Z T P_{F_{1}^{n}}, Z T P_{n}\right)=$ false

9: $\quad$ if $(N$ exist $)$ then

10: $\quad$ Explore $M s g . X=N$; update the routing table of $C$; forward Explore $M s g$ packet to the next-hop node $N$

11: else

\{no candidate node

send an Error Msg packet to the predecessor of $C$

end if

else if $\left(t<d_{c}\right)$ then

$\left\{\right.$ the first common node is at depth $\left.<d_{c}-1\right\}$

Explore $M$ sg. $X=P$; update the routing table of $C$; forward Explore $M s g$ packet to the next-hop node $P$ 18: end if

19: end if which is the first child of the Sink node (PAN)). As shown in the figure, three node disjoint paths are established from the source node $s$ to the Sink node. The first one is the classical path based on parent-child links (no discovery phase) and is the shortest one $P 1=s \rightarrow a \rightarrow$ Sink. To construct the second path, node $s$ takes node $c$ as its next-hop node. Since the intermediate node $c$ with $Z T P_{c}=(3,4)$ is a $N T R$ neighbor of the node $s(3 \neq 1$ and the $T R$ routing is not yet applied on the branch of $c$ ), consequently, $T R$ is applied from node $c$ until the destination node Sink and gives the second path $P 2=s \rightarrow c \rightarrow e \rightarrow$ Sink which is established automatically without triggering the discovery process. The node $s$ takes the node $k$ at depth $d_{k}=2$ as the next-hop node to build the third node disjoint path. Since $k$ is not a NTR neighbor of node $s$ (since $c$ and $k$ belong to the same branch, so $T R$ is already applied in this branch by node $c$ ), this case requires then a path discovery. The intermediate node $k$ compares its $Z T P_{k}=(3,2)$ with the node $c$ one $Z T P_{c}=(3,4)$ (the node which has firstly used the branch of the node $k$ ). It finds that the first commun node is at depth 1 (it is the node $e$ ) and can't be taken as the next-hop node (it already belongs to the path $P 2$ ). The node $k$ searches another candidate from its neighbors table. Since it can't choose the node $c$ because it belongs to the path $P 2$ (thanks to the inclusion operation) so it takes $y$ as its next-hop node. The node $y$ chooses the node $z$, which is its NTR neighbor, as the next-hop node. The node $z$ applied $T R$ until the Sink. The resulting node disjoint path is $P 3=s \rightarrow k \rightarrow y \rightarrow z \rightarrow t \rightarrow$ Sink.

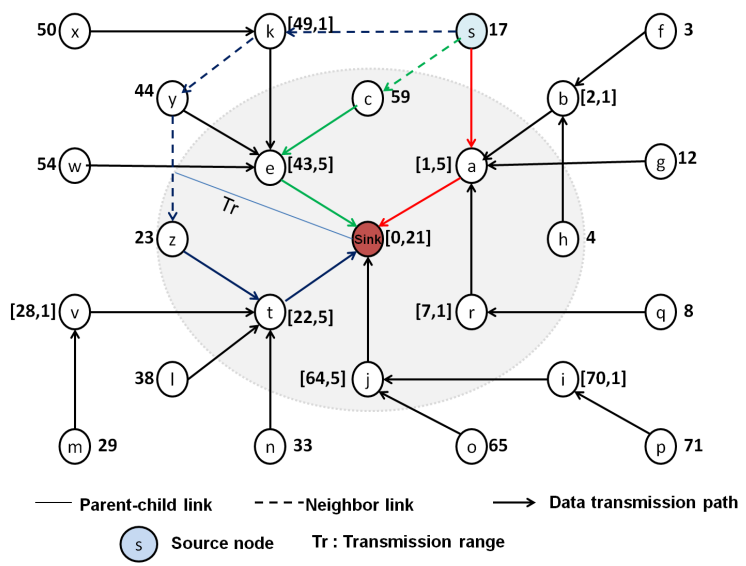

Figure 1. Example of node disjoint paths discovery from the source node $s$ to the Sink

\section{Simulation RESUlTS}

Different simulations have been carried out to evaluate the performances of the proposed ZigBee multipath routing protocol $Z M R$ with respect to the classical single ZigBee tree routing $(T R)$. Simulations are conducted using NS2 simulator with the IEEE 802.15.4 implementation provided by Zheng [28]. Even if $Z M R$ is able to build more than two 
paths, we limited our simulations to using only two paths simultaneously. It has been shown that using more than two paths does not achieve further throughput gain. The number of nodes in the network topology is fixed to 101 stationary nodes with one sink (the PAN coordinator) at the center of the area and 100 FFD nodes. The communication range is of $11 \mathrm{~m}$. The ZigBee Parameters $L_{m}, C_{m}$ and $R_{m}$ are 7,4 and 4 respectively. The non-beacon mode with unslotted CSMA/CA mechanism of the IEEE 802.15.4 standard is used at the MAC level. Propagation model used was two-ray ground model. The size of the packets payload is 80 bytes. Poisson traffic model is considered in our simulation and the traffic load is varied from 1 pps ( $0.625 \mathrm{kbps})$ to $100 \mathrm{pps}(62.5 \mathrm{kbps})$ to evaluate different metrics for different routing strategies.

Each scenario is simulated 12 times and the total number of simulation runs is $96(12 \times 8)$. Results over all the network scenarios are averaged to produce the packet delivery fraction, throughput, average end-to-end delay and network lifetime as a function of the traffic load.

Packet Delivery Fraction: Figure 2 shows that when the traffic load is in the range of 1 to 20pps all protocols have approximately the same behavior, they are able to handle the traffic quite well, delivering more than $99.5 \%$ of the packets to the destination. However, when the traffic load exceeds $20 p p s$, the multipath routing $Z M R$ outperforms the single routing $T R$ due to the insufficient bandwidth capacity of $T R$ to support such traffic load over one path and to the fact that the packet loss due to the intra-path collisions and interferences becomes higher for a single path.

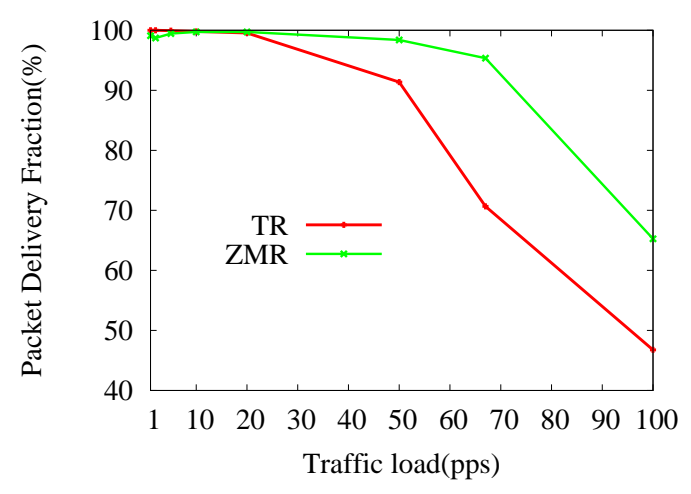

Figure 2. Packet delivery fraction in the different routing strategies

Average end to end delay: The average end to end packet delay, depicted by Figure 3 increases with the data rate since packets incur more queuing delay. Moreover, due to MAC collisions and interference, packets need to be retransmitted and so their delivery time increases. However, in the multipath routing, where the data packets are spread onto two node disjoint paths, the number of intra-path collisions and intrapath interference is reduced leading to less retransmissions and hence improve the end to end delay.

Network lifetime: The network is considered as not operational when the Sink stops receiving packets. Figure 4 shows

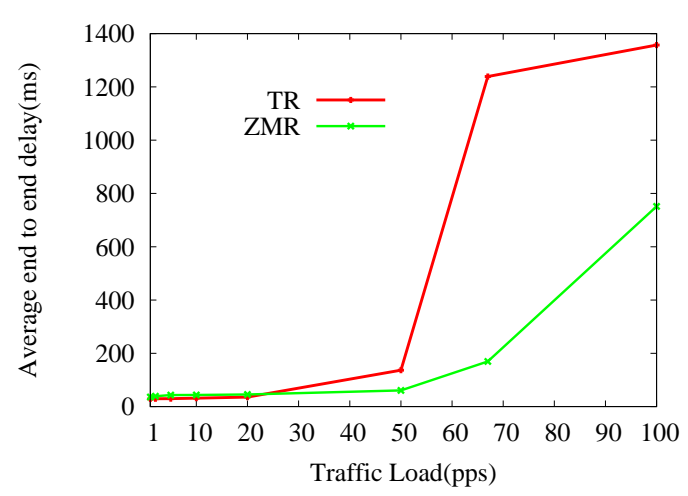

Figure 3. Average end to end delay in the different routing strategies

that the use of more than one path prolongs the network lifetime and hence gives better throughput performance. Beyond the saturation value 20pps, $T R$ suffers from excessive retransmissions (the entire traffic load would route along only one path), greatly degrading its performance, while the multipath routing (total network load is distributed evenly into two paths), can still enjoy the integrated capacity of multiple paths and achieves longer network lifetime.

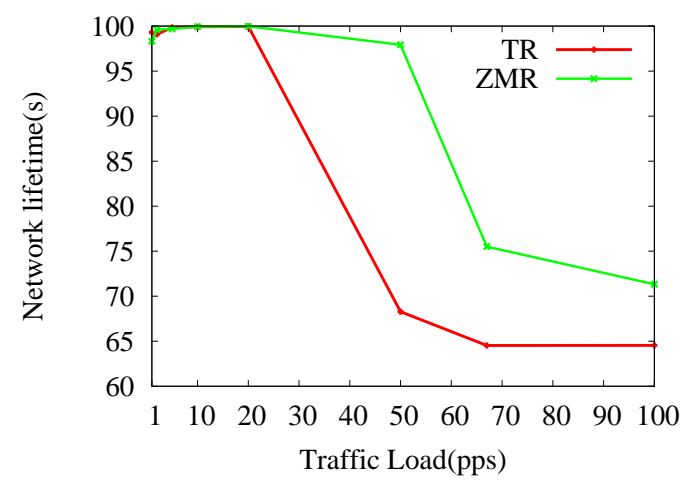

Figure 4. Network lifetime in the different routing strategies

Effective Bandwidth: Figure 5 shows that the multipath routing $Z M R$ outperforms the single path $T R$. To calculate the effective bandwidth of the $Z M R$ strategie, we need to consider the maximum throughput achievable by this technology (theoretically support $250 \mathrm{kbps}$ at $2.4 \mathrm{GHz}$ ). The simulation results of figure 5 indicate a maximum of throughput of $40756 b p s \simeq 41 k b p s$ is achievable when operating at a rate of $67 p p s \simeq 42 \mathrm{kbps}$ under this technology (This is equivalent to $1 / 6$ of the total bandwidth). However, utilizing this bandwidth is possible if we expect a QoS of $96 \%$ delivery of data. Therefore, if we expect a QoS of atleast $99 \%$ delivery of data, the throughput at lower datarates need to be considered. Thus referring to graphs 5 and 2, to achieve a delivery ratio of $99 \%$, the traffic rate should be $=50$ pps $(\simeq 31 \mathrm{kbps})$ and the achievable throughput at this rate of operation is $\simeq 31 \mathrm{kbps}$. However, in the case of the single path routing $T R$, the maximum throughput achievable is about $12.5 \mathrm{kbps}$ (equivalent 
to $1 / 18$ of the total bandwidth) for a QoS of $99 \%$ delivery of data expected, which is very insufficient for handling high data rate applications.

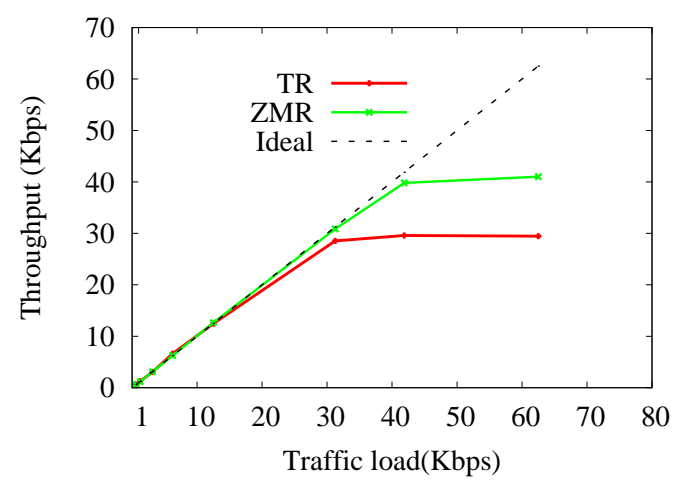

Figure 5. Throughput in the different routing strategies

\section{CONClusion}

In this paper, we proposed $Z M R$, a hybrid node disjoint multipath routing for ZigBee WSNs to handle high data rate applications. The proposed $Z M R$ protocol combines the proactive and the reactive approaches in order to construct multiple node disjoint paths. For this aim, it exploits judiciously the address assignment scheme of ZigBee and the parent-child and non parent-child links. Results showed that the multipath routing $Z M R$ provides the best performances in terms of packet delivery fraction, end to end delay, network lifetime, throughput and bandwidth with regard to the single path routing $T R$ and satisfies soft QoS provision requirements of the high traffic applications.

\section{REFERENCES}

[1] T. Wark, P. Corke, P. Sikka, L. Klingbeila, Y. Guo, C. Crossman, P. Valencia, D. Swain, and G. Bishop-Hurley, "Transforming agriculture through pervasive wireless sensor networks," IEEE Pervasive Computing, vol. 6, no. 2, pp. 50-57, 2007.

[2] A. Mainwaring, D. Culler, J. Polastre, R. Szewczyk, and J. Anderson, "Wireless sensor networks for habitat monitoring," in Proceedings of the 1st ACM International Workshop on Wireless Sensor Networks and Applications, ACM, New york, USA, 2002, pp. 88-97.

[3] A. Milenkovic, C. Otto, and E. Jovanov, "Wireless sensor networks for personal health monitoring: issues and an implementation," Computer Communications, vol. 29, no. 13-14, pp. 2521-2533, 2006.

[4] V. Gungor and G. Hancke, "Industrial wireless sensor networks: challenges, design principles, and technical approaches," IEEE Transactions on Industrial Electronics, vol. 56, no. 10, pp. 4258-4265, 2009.

[5] "Waspmote video camera, technical guide," 2013, http://www.libelium.com.

[6] "CSIRO multimedia node," http://www.sensornets.csiro.au.

[7] I. F. Akyildiz, T. Melodia, and K. R. Chowdhury, "A survey on wireless multimedia sensor networks," Computer Networks, vol. 51, no. 4, pp. 921-960, March 2007. [Online]. Available: http://dx.doi.org/10.1016/j.comnet.2006.10.002

[8] I. Akylidiz, Y. S. W. Su, and E. Cayirci, "Wireless sensor networks: A survey," Computer Networks, vol. 38, no. 4, pp. 393-422, December 2002.

[9] F.-S. A.J.Garcia-Sanchez and J.Garcia-Haro, "Feasibility study of mpeg4 transmission on ieee 802.15.4 network," in Networking and Communications, 2008. WIMOB'08. IEEE International Conference on Wireless and Mobile Computing, Avignon, October 2008, pp. 397-403.
[10] S.Deshpande, "Adaptive low-bitrate streaming over ieee 802.15.4 low rate wireless personal area networks (lr-wpan) based on link quality indication," in International Conference On Communication and Mobile Computing, Vancouver, Canada, 2006, pp. 863-868.

[11] F.-S. A.J.Garcia-Sanchez, J.Garcia-Haro, and F.Losilla, "A cross-layer solution for enabling real-time video transmission over ieee 802.15.4 networks, published online," Multimedia Tools and Applications, vol. 51, no. 3, pp. 1069-1104, February 2011.

[12] F.QIN, "Technologies to improve the performance of wireless sensor networks in high-traffic applications," Ph.D. dissertation, Department of Electronic and Electrical Engineering University College London, 2012.

[13] "Zigbee alliance. zigbee specification document 053474r17," January 2008.

[14] N. T.Kim, D.Kim, S.Yoo, and T.S.Lopez, "Shortcut tree routing in zigbee networks," in 2nd International Symposium Wireless Pervasive Computing, 2007.

[15] B.Nefzi and Y.Q.Song, "performance analysis and improvement of zigbee routing protocol," in 7th IFAC International Conference on Fieldbuses \& Networks in Industrial \& Embedded Systems - FeT'2007, Toulouse France, 2007.

[16] S. J.Y.Ha, H.S.Park and W.H.Kwon, "EHRP: Enhanced hierarchical routing protocol for zigbee mesh networks," in IEEE Communications Letters, vol. 11, 2007, pp. 1028-1030.

[17] Y. C.H.Tsai, M.S.Pan and Y.C.Tseng, "Self-learning routing for zigbee wireless mesh networks," 2009.

[18] S. S. D. Ganesan, R. Govindan and D. Estrin, "Highly-resilient, energyefficient multipath routing in wireless sensor networks," Mobile Computing and Communication Review, vol. 5, no. 4, pp. 10-24, 2001

[19] S. Wan and Y. He, "K-multipath routing mechanism with load balancing in wireless sensor networks," in Proceedings of the Second Symposium International Computer Science and Computational Technology(ISCSCT'09), Huangshan, P. R. China, 26-28 December 2009, pp. $385-388$.

[20] X. Huang and Y. Fang, "End-to-end delay differentiation by prioritized multipath routing in wireless sensor networks." in Military Communications Conference (MILCOM), Atlantic City, 2005, pp. 1277-1283.

[21] Z.Bidai, H.Haffaf, and M.Maimour, "Node disjoint multi-path routing for zigbee cluster tree wireless sensor networks," in International Conference on Multimedia Computing and Systems (ICMCS 2011), Ouarzazate, Morocco, 7-9 April 2011.

[22] Z.Bidai, M.Maimour, and H.Haffaf, "Multipath extension of the zigbee tree routing in cluster-tree wireless sensor networks," International Journal of mobile Computing and Multimedia Communications (IJMCM), vol. 4, no. 2, 2012.

[23] "IEEE-TG15.4 standards part 15.4: Wireless medium access control (MAC) and physical layer (PHY) specifications for low-rate wireless personal area networks (LR-WPANs)," September 2006.

[24] P. Baronti, P. Pillai, V. W. C. Chook, S. Chessa, A. Gotta, and Y. F. $\mathrm{Hu}$, "Wireless sensor networks: A survey on the state of the art and the 802.15.4 and zigbee standards," Comput. Commun., vol. 30, no. 7, pp. $1655-1695,2007$.

[25] C. Perkins, E. Royer, and S. Das, "Ad hoc on demand distance vector (aodv) routing," 1999. [Online]. Available: http://www.ietf.org/internet-drafts/draft-ieftmanet-aodv-03.txt

[26] R. Karthikeyan, "Routing strategy selection for zigbee mesh networks," International Journal Communications . Network and System Sciences, vol. 3, pp. 608-611, 2010.

[27] P. Ran, M. Sun, and Y. Zou, "Zigbee routing selection strategy based on data services and energy balanced zigbee routing," in In Proceedings of the IEEE Asia-Pacific Conference on Services Computing, 2006, pp. 400-404.

[28] M. Zheng, "NS2 simulator for IEEE 802.15.4," 2004, url http://ees2cy.engr.ccny.cuny.edu/zheng/pub/. 